\title{
THE RING OF HOLOMORPHIC FUNCTIONS ON A STEIN COMPACT SET AS A UNIQUE FACTORIZATION DOMAIN
}

\author{
H. G. DALES
}

\begin{abstract}
Let $\Gamma$ be the ring of germs of analytic functions on a Stein compact subset $K$ of a complex-analytic space. Necessary and sufficient conditions on $K$ for $\Gamma$ to be a unique factorization domain are given.
\end{abstract}

Let $X$ be a complex-analytic space with structure sheaf $\mathcal{O}$, and let $K$ be a compact Stein subset of $X$, so that $K$ has a neighbourhood base in $X$ consisting of Stein open subsets of $X$. Let $\Gamma=\Gamma(K, \mathcal{O})$, the ring of germs of analytic functions on $K$. In this note, we determine necessary and sufficient conditions for $\Gamma$ to be a unique factorization domain.

Conditions for $\Gamma$ to be a Noetherian ring have been given by Siu [5], following an earlier result of Frisch [2]:

THEOREM (SiU). Let $K$ be a compact Stein subset of an analytic space $(X, \mathcal{O})$. Then $\Gamma(K, \mathcal{O})$ is Noetherian if and only if $V \cap K$ has only finitely many topological components for each complex-analytic subvariety $V$ defined in an open neighbourhood of $K$.

If $z$ is a point of an arbitrary complex-analytic space, then $\mathcal{O}_{z}$, the ring of analytic function germs at $z$, is not necessarily a unique factorization domain, for, if it is, then $z$ is a normal point of $X$. On the other hand, if $z$ is a simple point of $X$, which means that $\mathcal{O}_{z}$ is a regular local ring, or, equivalently, that a neighbourhood of $z$ in $X$ can be mapped bianalytically onto an open set in $\boldsymbol{C}^{n}$, then $\mathcal{O}_{z}$ is a unique factorization domain by $[4, \S 4.2]$. We shall suppose explicitly that $\mathcal{O}_{z}$ is a unique factorization domain for each $z$ in $K$. Thus, the hypotheses on $K$ are satisfied if $K$ is a compact subset of a complex manifold $X$ and $K$ is the intersection of a sequence of open Stein manifolds (i.e., $K$ is a holomorphic set).

We shall require Cartan's theorems A and B for coherent analytic sheaves over the compact set $K$. These theorems are stated for the context in which we require them in $[1$, Théorème 1$]$.

Received by the editors April 10, 1973 and, in revised form, July 27, 1973.

AMS (MOS) subject classifications (1970). Primary 32E25; Secondary 13E05, 13 F15.

Key words and phrases. Analytic space, germs of analytic functions, Stein compact set, Noetherian ring, unique factorization domain.

(c) American Mathematical Society 1974 
Let $\mathscr{M}$ denote the sheaf of meromorphic functions on $K$, and let $\mathcal{O}^{*}$ denote the sheaf of invertible elements in $\mathcal{O}$ (with multiplication as group operation). We say that $K$ is a Cousin II set if the second Cousin problem can be solved on $K$ : if $\left\{U_{\alpha}\right\}$ is a cover of $K$ consisting of (relatively) open subsets, and if $f_{\alpha} \in \Gamma\left(U_{\alpha}, \mathcal{O}\right)$ such that $f_{\alpha} f_{\beta}^{-1} \in \Gamma\left(U_{\alpha} \cap U_{\beta}, \mathcal{O}^{*}\right)$ for each $\alpha, \beta$, then there exists $f \in \Gamma(K, \mathcal{O})$ such that $f f_{\alpha}^{-1} \in \Gamma\left(U_{\alpha}, \mathcal{O}^{*}\right)$ for each $\alpha$. The set $K$ is a strong Poincaré set if, given $m \in \Gamma(K, \mathscr{M})$, there exist $f, g \in \Gamma(K, \mathcal{O})$ such that $m=f g^{-1}$ and $\left(f_{z}, g_{z}\right)=1_{z}$ in $\mathcal{O}_{z}(z \in K)$ (i.e., the germs $f_{z}$ and $g_{z}$ are coprime for each $z$ ). Let $I$ be an ideal in $\Gamma(K, \mathcal{O})$, and let $\mathscr{I}$ be the sheaf of ideals of $\mathcal{O} \mid K$ generated over $K$ by $I$. Then we say that $I$ is a locally principal ideal in $\Gamma$ if $\mathscr{I}_{z}$ is a principal ideal in $\mathcal{O}_{z}$ for each $z \in K$.

THEOREM 1. Let $K$ be a compact Stein subset of a complex-analytic space $X$ with structure sheaf $\mathcal{O}$, and suppose $\mathcal{O}_{z}$ is a unique factorization domain for each $z \in K$. Suppose that $\Gamma=\Gamma(K, \mathcal{O})$ is a Noetherian domain. Then the following conditions are equiralent:

(1) $\Gamma$ is a unique factorization domain;

(2) $H^{2}(K ; Z)=\{0\}$ :

(3) $K$ is a Cousin II set;

(4) $K$ is a strong Poincaré set;

(5) every locally principal ideal in $\Gamma$ is a principal ideal.

Proof. We first prove the implications which do not require the supposition that $\Gamma$ be a Noetherian ring.

$(2) \Leftrightarrow(3) \Rightarrow(4)$. Using Cartan's Theorems A and B, these are standard results (cf. [3, VIII, B]).

(5) $\Rightarrow(3)$. Let $\left\{f_{\alpha} ; U_{\alpha}\right\}$ be data for the second Cousin problem on $K$. Define the sheaf $\mathscr{F}$ over $K$ by the conditions $\mathscr{F}_{z}=\left\langle f_{\alpha, z}\right\rangle\left(z \in U_{\alpha}\right)$. Then $\mathscr{F}_{z}$ is well defined, for $\left\langle f_{\alpha, z}\right\rangle=\left\langle f_{\beta, z}\right\rangle$ whenever $z \in U_{\alpha} \cap U_{\beta}$, and $\mathscr{F}$ is a coherent analytic sheaf over $K$. Let $I=\Gamma(K, \mathscr{F})$ be the algebra of sections of $\mathscr{F}$ over $K$, and let $I$ generate $\mathscr{I}$ in $\mathcal{O} \mid K$. By Theorem A, $\mathscr{I}_{z}=\mathscr{F}_{z}$ for $z \in K$, so that $I$ is a locally principal ideal in $\Gamma$. By hypothesis, $I$ is a principal ideal in $\Gamma$, say $I=\langle f\rangle$. Clearly, $f$ is a solution of the problem with data $\left\{f_{\alpha} ; U_{\alpha}\right\}$.

$(1) \Rightarrow(4)$. Let $m \in \Gamma(K, \mathscr{M})$. Since $K$ is a Stein set, we can write $m=f_{1} g_{1}^{-1}$ with $f_{1} g_{1} \in \Gamma$ (cf. [3, VIII, B, 10]), and, by Theorem A again, we can suppose that $\left(f_{1, z}, g_{1, z}\right)=1_{z}$ for some fixed $z \in K$. Clearly, using the hypothesis, we can also suppose that $\left(f_{1}, g_{1}\right)=1$ in $\Gamma$. Let $w^{\prime}$ be any point of $K$, and write $m=f_{2} g_{2}^{-1}$ with $f_{2}, g_{2} \in \Gamma,\left(f_{2}, g_{2}\right)=1$ in $\Gamma$, and $\left(f_{2, w}, g_{2, w}\right)=$ $1_{w}$. Then $f_{1} g_{2}=f_{2} g_{1}$ in $\Gamma$, and, since $\Gamma$ is a unique factorization domain, $f_{1} \sim f_{2}$ and $g_{1} \sim g_{2}$ in $\Gamma$. Thus, $\left(f_{1, w}, g_{1, w}\right)=\left(f_{2, w}, g_{2, w}\right)=1_{w}$ in $\mathcal{O}_{w}$ for each 
$w \in K$. It follows that $f_{1}$ and $g_{1}$ are coprime at each point of $K$, and we have written $m=f_{1} g_{1}^{-1}$ in the required form.

In the proof of the remaining implications, we use the fact that $\Gamma$ is a Noetherian domain.

$(4) \Rightarrow(1)$. Since $\Gamma$ is Noetherian, it is sufficient to prove that every irreducible element in $\Gamma$ is prime. Let $f$ be an irreducible in $\Gamma$, and suppose that $g h \in\langle f\rangle$, say $g h=f f_{1}$ in $\Gamma$. Since $K$ is a strong Poincaré set and $\Gamma$ is an integral domain, we may suppose that $\left(g_{z}, f_{1, z}\right)=1_{z}$ in $\mathcal{O}_{z}(z \in K)$. Since $g_{z} h_{z}=f_{z} f_{1, z}, h_{z} \in\left\langle f_{1, z}\right\rangle(z \in K)$, and so, from Theorem B, $h \in\left\langle f_{1}\right\rangle$ in $\Gamma$, say $h=f_{1} h_{1}$. Thus, $g h_{1}=f$, and since $f$ is irreducible, either $g$ or $h_{1}$ is a unit in $\Gamma$. In the former case, $h \in\langle f\rangle$, and in the latter, $g \in\langle f\rangle$, so that $f$ is a prime, as required.

$(1) \Rightarrow(5)$. Let $I$ be a locally principal ideal in $\Gamma$, and let $\mathscr{I}$ be as above. Since $I$ is finitely generated, $\mathscr{I}$ is a coherent analytic sheaf over $K$.

Let $z \in K$. By hypothesis, $\mathscr{I}_{z}$ is a principal ideal in $\mathcal{O}_{z}$, and so, by Theorem A, there exists $f \in I$ such that $\mathscr{I}_{z}=\left\langle f_{z}\right\rangle$ in $\mathscr{O}_{z}$. Since $K$ is compact, there exist $f_{1}, \cdots, f_{k} \in I$ such that, for each $z \in K, \mathscr{I}_{z}=\left\langle f_{i, z}\right\rangle$ for some $i=i(z) \in\{1, \cdots, k\}$. By Theorem $\mathbf{B}, I=\left\langle f_{1}, \cdots, f_{k}\right\rangle$. Since $\Gamma$ is a unique factorization domain, $f_{1}, \cdots, f_{k}$ have a highest common factor in $\Gamma$, say $g=\left(f_{1}, \cdots, f_{k}\right)$. Let $h_{i}=f_{i} g^{-1}(i=1, \cdots, k)$, so that $\left(h_{1}, \cdots, h_{k}\right)=1$.

We now use hypothesis (1) to prove that, if $\left(p_{1}, \cdots, p_{n}\right)=1$ in $\Gamma$, then $\left(p_{1, z}, \cdots, p_{n, z}\right)=1_{z}$ in $\mathcal{O}_{z}(z \in K)$. The result holds for the case $n=2$ by the result ' $(1) \Rightarrow(4)$ ', above, and the general result follows by an immediate inductive argument. Thus, we see that we have $h_{1}, \cdots, h_{k} \in \Gamma$ with

$$
\left(h_{1, z}, \cdots, h_{k, z}\right)=1_{z} \quad \text { in } \mathcal{O}_{z}(z \in K) \text {. }
$$

Take $z \in K$, and suppose that $\mathscr{I}_{z}=\left\langle f_{j, z}\right\rangle$. If $i \in\{1, \cdots, k\}$, there exists $p_{i, z} \in \mathcal{O}_{z}$ such that $f_{i, z}=p_{i, z} f_{j, z}$. Thus, $h_{i, z}=p_{i, z} h_{j, z}$ and so $h_{j, z} \mid h_{i, z}$ in $\mathcal{O}_{z}(i \in\{1, \cdots, k\})$. This shows that $h_{j, z} \mid\left(h_{1, z}, \cdots, h_{k, z}\right)$ in $\mathcal{O}_{z}$. From $(*), h_{j, z}$ is a unit in $\mathcal{O}_{z}$, and we have proved that the functions $h_{1}, \cdots, h_{k}$ have no common zero on $K$. It is a consequence of Cartan's Theorem B [3, VIII, A, 16] that there exist $p_{1}, \cdots, p_{k} \in \Gamma$ such that $\sum p_{i} h_{i}=1$. Hence, $g=\sum p_{i} g f_{i} \in I, I=\langle g\rangle$, and $I$ is a principal ideal in $\Gamma$, as required.

This concludes the proof of the theorem.

Suppose that $\Gamma$ is a regular Noetherian domain in the sense of Kaplansky [4]. Then the following proof that ' $(5) \Rightarrow(1)$ ' holds.

Let $I$ be an ideal in $\Gamma$ generating the coherent sheaf $\mathscr{I}$ in $\mathcal{O} \mid K$. Suppose that $I$ is invertible $\left[4\right.$, p. 37] in $\Gamma$. We first note that $\mathscr{I}_{z}$ is invertible in $\mathcal{O}_{z}(z \in K)$. We must prove that $\mathcal{O}_{z} \subset \mathscr{I}_{z} \mathscr{I}_{z}^{-1}$, so take $f_{z} \in \mathcal{O}_{z}$. Then $f_{z} \in \sum f_{i, z} \mathcal{O}_{z}$ with $f_{i} \in \Gamma$. Since $I$ is invertible, there exist $g_{i j} \in I$ and $h_{i j} \in I^{-1}$ such that $f_{i}=\sum_{j} g_{i j} h_{i j}$ for each $i$. Thus, $f_{z} \in \sum_{i, j} g_{i j, z} h_{i j, z} \mathcal{O}_{z}$. Clearly, $g_{i j, z} \in \mathscr{I}_{z}$. Also, $h_{i j, z} \in \mathscr{I}_{z}^{-1}$, for, if $p_{z} \in \mathscr{I}_{z}$, then, by Theorem A, 
$p_{z} \in \sum q_{k, z} \mathcal{O}_{z}$ with $q_{k} \in I$, and, since $I$ is invertible, $h_{i j}\left(\sum q_{k} \Gamma\right) \subset \Gamma$, so that $h_{i j, z} p_{z} \in \mathcal{O}_{z}$ and $h_{i j, z} \in \mathscr{I}_{z}^{-1}$, as required. Thus, $\mathcal{O}_{z} \subset \mathscr{I}_{z} \mathscr{I}_{z}^{-1}$.

Now, by [4, Theorem 60], $\mathscr{I}_{z}$ is principal in $\mathcal{O}_{z}(z \in K)$, so, by hypothesis, $I$ is principal in $\Gamma$, and the result follows by [4, Theorem 185].

If $K$ is a compact, holomorphic set in $C^{n}$ for which $\Gamma$ is a Noetherian domain, then $\Gamma$ is a regular Noetherian domain. To show this, it suffices to show that every maximal ideal of $\Gamma$ can be generated by an $R$-sequence [4, §3.1]. But if $M$ is a maximal ideal of $\Gamma$, then $M=\left\{f \in \Gamma: f\left(z^{0}\right)=0\right\}$ for some $z^{0} \in K$, and it is clear that the elements $z_{1}-z_{1}^{0}, \cdots, z_{n}-z_{n}^{0}$ form the required $R$-sequence.

Corollary. Let $\Delta$ be a compact polydisc in $C^{n}$. Then $\Gamma(\Delta, \mathcal{O})$ is a unique factorization domain.

Proof. That $\Gamma(\Delta, \mathcal{O})$ is a Noetherian domain is noted in [2]. Certainly, $H^{2}(\Delta ; Z)=\{0\}$.

When $\Gamma=\Gamma(K, \mathcal{O})$ is Noetherian, we have the following Nullstellensatz for $\Gamma$. We use the notation of $[3, I I, E]$.

Proposition 2. Let $K$ be a compact Stein subset of a complex-analytic space $X$, and suppose that $\Gamma$ is a Noetherian domain. Let I be an ideal in $\Gamma$. Then id loc $I=\operatorname{rad} I$.

Proof. Certainly, rad $I \subset$ id loc $I$. Take $f \in$ id loc $I$. If $z \in K$, then there exists $k(z) \in N$ such that $f_{z}^{k(z)} \in \mathscr{I}_{z}$. This is the standard Nullstellensatz, proved in the local complex-analytic case in [3, III. A. 7]; the general case follows by writing $\mathscr{I}$ locally as a quotient of an ideal sheaf in $\boldsymbol{C}^{n}$ [6, Lemma]. For $w$ in a neighbourhood of $z, f_{w}^{k(z)} \in \mathscr{I}_{w}$. Take a finite refinement of the neighbourhoods covering $K$, corresponding to $z_{1}, \cdots$, $z_{m}$, and let $k=\max \left\{k\left(z_{1}\right), \cdots, k\left(z_{m}\right)\right\}$. Then $f_{z}^{k} \in \mathscr{I}_{z}(z \in K)$, and, since $\mathscr{I}$ is coherent, $f^{k} \in I$. Thus, id $\operatorname{loc} I \subset \operatorname{rad} I$, as required.

Let $f \in \Gamma$, and write $V(f)=\{z \in K: f(z)=0\}$. A variety $V$ is irreducible if $V=V_{1} \cup V_{2}$, where $V_{1}$ and $V_{2}$ are varieties, implies that either $V=V_{1}$ or $V=V_{\mathrm{z}}$.

Theorem 3. Let $K$ and $X$ be as in Theorem 1. Suppose that $\Gamma$ is a Noetherian domain which is a unique factorization domain, and let $f \in \Gamma$.

(i) $V(f)$ is an irreducible variety if and only if $f=g^{n}$, where $g$ is irreducible in $\Gamma$.

(ii) If $f=\prod_{i=1}^{n} f_{i}^{k_{i}}$ is the factorization of $f$ into irreducible factors in $\Gamma$, then $V(f)=\bigcup_{i=1}^{n} V\left(f_{i}\right)$ is the decomposition of $V$ into its irreducible branches.

Proof. If $f \in \Gamma$ is irreducible, then $\langle f\rangle$ is prime, and so, by Proposition 2 , id $V(f)$ is prime. The results now follow by the same arguments as those of $[3, I I, E]$. 
I am grateful to Dr. P. F. Smith and Professor E. L. Stout for valuable conversations on the above results.

\section{REFERENCES}

1. H. Cartan, Variétés analytiques réelles et variétés analytiques complexes, Bull. Soc. Math. France 85 (1957), 77-99. MR 20 \#1339.

2. J. Frisch, Points de platitude d'un morphisme d'espaces analytiques complexes, Invent. Math. 4 (1967), 118-138. MR 36 \#5388.

3. R. C. Gunning and H. Rossi, Analytic functions of several complex variables, Prentice-Hall Series in Modern Analysis, Prentice-Hall, Englewood Cliffs, N.J., 1965. MR 31 \#4927.

4. I. Kaplansky, Commutative rings, Allyn and Bacon, Boston, Mass., 1970. MR 40 \#7234.

5. Y.-T. Siu, Noetherianness of rings of holomorphic functions on Stein compact subsets, Proc. Amer. Math. Soc. 21 (1969), 483-489. MR 40 \#404.

6. - Hilbert Nullstellensatz in global complex-analytic case, Proc. Amer. Math. Soc. 19 (1968), 296-298. MR 36 \#5391.

Department of Mathematics, University of Glasgow, Glasgow, G12 8QW SCOTLAND

Current address: Department of Mathematics, University of California, Los Angeles, California 90024 